Association between Decentering and Reductions in Relapse/Recurrence in Mindfulness-Based Cognitive Therapy for Depression in Adults: A Randomized Controlled Trial

Michael T. Moore ${ }^{1}$, Mark A. Lau ${ }^{2}$, Emily A.P. Haigh ${ }^{3}$, Brandilyn R. Willett ${ }^{2}$, Colin M. Bosma ${ }^{3}$ \& David M. Fresco ${ }^{4}$

\author{
${ }^{1}$ Adelphi University \\ ${ }^{2}$ University of British Columbia \\ ${ }^{3}$ University of Maine \\ ${ }^{4}$ University of Michigan
}

\begin{abstract}
Authors Note
We have no known conflicts of interest to disclose.

Dr. Lau was supported by CIHR Grant 190696. Dr. Fresco was supported by NHLBI Grant R01HL119977, NCCIH Grant R61AT009867, NIMH Grant R01MH118218, NICHD Grant R21HD095099 and NCI Grant R01CA244185.

All data, study materials, and analysis code is available from the first author, by request.

Because the trial was conceived prior to 2008, pre-registration was not possible. However, the trial was registered on ClinicalTrials.gov after data collection and analysis (Title: "Decentering and Relapse/Recurrence in MBCT for Depression in Adults”, Identifier: NCT05111665).
\end{abstract}

Corresponding Author: $\quad$ Michael T. Moore

Adelphi University

Gordon F. Derner School of Psychology

Blodgett Hall, $2^{\text {nd }}$ Floor

Garden City, NY 11530

Telephone: (516) 877-4744

E-mail: mmoore@adelphi.edu 
Association between Decentering and Reductions in Relapse/Recurrence in Mindfulness-Based Cognitive Therapy for Depression in Adults: A Randomized Controlled Trial

Submitted: 11/9/2021

Resubmitted: 1/27/2022 


\begin{abstract}
Objective: "Decentering” is defined as the ability to observe one’s thoughts and feelings as temporary, objective events in the mind (Safran \& Segal, 1990), and is increasingly regarded as a candidate mechanism in mindfulness-based interventions. The current study sought to examine the role of decentering, and other related variables, in the efficacy of Mindfulness-based cognitive therapy (MBCT) as compared to two active comparison conditions.
\end{abstract}

Method: Formerly depressed individuals $(N=227)$, randomly assigned to $\operatorname{MBCT}(n=74)$, relaxation group therapy (RGT; $n=77$ ) or treatment-as-usual (TAU; $n=76$ ), completed selfreport measures of decentering and symptoms of depression at pre-, mid-, and post-treatment, and relapse was assessed at 3, 6, 9, and 12 months, post-treatment.

Results: With regard to the acute treatment phase, results indicated that, whereas levels of depression increased in both RGT and TAU, MBCT patients remained free from symptom gains. Moreover, gains in decentering from mid- to post-treatment predicted reductions in depression from pre- to post-treatment for MBCT and TAU, but not for RGT. Participants who experienced increases in decentering, measured from mid- to post-treatment, generally evidenced the lowest levels of relapse/recurrence (during the four follow-up assessments), largely irrespective of treatment group. However, results related to change in decentering should be considered exploratory due to small cell sizes among participants who did not experience gains in decentering.

Conclusions: Taken together, these results suggest that decentering is a potent mechanism for reduction of relapse in major depression, albeit one that is non-specific to MBCT. 
Keywords: decentering, mindfulness-based cognitive therapy, major depression, relapse prevention, relaxation group therapy

Public Health Significance Statement: Gains in decentering, regardless of treatment modality, are associated with reductions in relapse/recurrence in depression.

Data Transparency Statement: The variables and relationships examined in the present article have not been examined in any previous or current articles, or to the best of our knowledge in any papers that will be under review soon. All data and syntax are available from the first author upon request. 


\section{Association between Decentering and Reductions in Relapse/Recurrence in Mindfulness- Based Cognitive Therapy for Depression in Adults: A Randomized Controlled Trial}

Depression is a common and debilitating form of mental illness with profound implications for the public_-affecting over 13 million individuals per year in the United States (Kessler et al., 2003), or about 10\% of the population during that time period, and may be increasing in prevalence over time (Weinberger et al., 2018). Depression is also a recurrent disorder, as a third of all individuals (sampled from a community setting) with one episode will have another (Eaton et al., 2008); this figure increases to three-quarters in individuals sampled from an outpatient mental health setting (Mueller et al., 1999). Thus, we face a public health necessity to identify and refine treatments for depression during both the acute phase, as well successfully preventing relapse (the return of symptoms associated with a remitted depressive episode) and recurrence (the onset of a new depressive episode following recovery). Various forms of cognitive-behavioral therapy (e.g., cognitive therapy, behavioral activation) are highly effective at treating acute depression to remission and reducing risk for relapse (see Cuijpers, Berking, Andersson, Quigley, Kleiboer, and Dobson, 2013, for a review). However, the one-year relapse rate for clients previously treated with cognitive therapy is approximately 25\%. This statistic indicates both the need for further refinement and room for improvement.

Given the prevalent (Kessler et al., 2003) and recurrent nature (Sim et al., 2015) of major depressive disorder (MDD), Mindfulness-Based Cognitive Therapy (MBCT; e.g., Segal, Williams, \& Teasdale, 2013) was designed (as a group treatment) to target a putative risk factor associated with relapse (i.e., mood-activated negative repetitive thought) by changing the way individuals relate to their thoughts (e.g., decentering) and cognitive restructuring. On balance, the available research (see Piet \& Hougaard, 2011 and Thimm \& Johnsen, 2020 for reviews) 
shows that MBCT is effective in reducing the relative risk of relapse/recurrence in MDD by $34 \%$, overall, when compared to placebo or treatment-as-usual (TAU). This reduction in risk is 43\% for participants with three or more prior major depressive episodes.

To date, the majority of randomized controlled trials have compared MBCT to no treatment, placebo, or antidepressant medication; very few trials have compared MBCT to an active psychosocial comparator (Shallcross et al., 2018). The literature comparing MBCT to an active treatment has, thus far, largely failed to identify MBCT as the superior intervention for all participants, with regard to reduction of relapse in MDD (see below for a review). The superiority of MBCT has, in most trials, only emerged among subgroups of participants. One possible explanation for this unexpected pattern of results may lie in the study of the mechanisms of MBCT. Although many studies have attempted to identify if MBCT is effective at reducing relapse in depression, relatively few studies have examined how MBCT works by looking at mechanisms of therapeutic action, and only two prior studies (Bieling et al., 2012; Segal et al., 2019) have investigated decentering as a possible mechanism. The current study seeks to contribute to the literature via comparison of MBCT to both an active psychosocial comparison condition (i.e., relaxation group therapy) and a waitlist comparison condition, and to examine decentering as a potential mechanism by which MBCT reduces risk for relapse/recurrence in MDD. Identification of mechanisms of action can potentially help to strengthen MBCTs effects by increasing the "dose” of these mechanisms and removing aspects of treatment that do not contribute to outcome. Identification of mechanisms can also potentially help to identify who will (and will not) benefit from MBCT and account for the aforementioned heterogeneity in results of MBCT trials using an active comparison condition.

\section{Efficacy of MBCT in Reducing Risk for Relapse/Recurrence of MDD}


Four prior MBCT trials, using treatment-as-usual (TAU) as the comparator, consistently found an advantage for MBCT in terms of greater reduction in risk for relapse/recurrence of MDD over a period of between 14 and 18 months, post-treatment (Bondolfi et al., 2010; Godfrin \& van Heeringen, 2010; Ma \& Teasdale, 2004; Teasdale, Williams, Ridgeway, Soulsby, \& Lau, 2000). Two RCTs compared MBCT to an active medication comparator (Kuyken et al., 2008; Segal et al., 2010). Kuyken et al. (2008) reported an advantage for MBCT, as compared to maintenance antidepressant medication (ADM) among MDD patients, treated to remission with ADM over 15 months of follow-up. Similarly, Segal et al. (2010) randomly assigned MDD patients treated to remission with ADM to one of three arms: MBCT, maintenance ADM, or a pill placebo group, who were then followed for 18 months. Unlike Kuyken et al. (2008), Segal et al. (2010) found relapse/recurrence rates only differed between pill placebo and the remaining groups and only for participants whose remission was defined as unstable (i.e., Hamilton Depression Rating Scale (Hamilton, 1960) > 7; Range 8-14).

Trials comparing MBCT to an active psychosocial comparator, equated for time and intensity, have not consistently found an advantage for MBCT (Farb et al., 2018; Meadows et al., 2014; Shallcross et al., 2018; Williams et al., 2014). For instance, no study has found MBCT superior to the active comparator for all participants. Rather, treatment superiority for MBCT emerged only when examining subgroups of participants. Meadows et al. (2014) compared MBCT to “depression relapse active monitoring (DRAM)”. Participants were randomized to either MBCT plus DRAM or to DRAM, alone. Findings indicated that MBCT plus DRAM was only associated with a greater time to relapse/recurrence for: (1) participants who received usual care in a specialist (as opposed to primary care) setting and (2) participants who were also taking antidepressant/mood stabilizer medication (this effect was, itself, only found in per protocol, not 
intent to treat, analysis). Given that DRAM involved less group and therapist contact, as compared to MBCT, this study leaves open the possible interpretation that differences between groups in psychotherapy “dose” affected the results of this trial. In addition, because MBCT was not administered by itself, the results could be interpreted as evidence that DRAM limited the efficacy of MBCT.

Williams et al. (2014) compared MBCT plus TAU to “cognitive psychological education (CPE)," an active comparator, plus TAU. CPE was designed to consist of "all elements of the MBCT program except the experiential cultivation of mindfulness,” (p. 278) and home meditation practice. Results indicated that MBCT was associated with less risk for relapse/recurrence only in participants above the median severity of childhood trauma. Due to the study design (that all participants received TAU), it is unclear if these results reflect a lack of therapeutic effect for MBCT and CPE or if the effects of TAU overlapped with these two treatments such that they washed-out the effects of both MBCT and CPE.

Shallcross et al. (2018) compared MBCT to Health Enhancement Program (HEP; MacCoon et al., 2012), itself a validated and manualized treatment that was designed to be equivalent to MBSR in group and facilitator contact and at home practice. HEP consisted of physical activity, nutritional advice, and music therapy, among other components. Shallcross et al. (2018) was also noteworthy for extending the follow-up period to 26 months. Results of this trial found no differences in risk of relapse/recurrence of MDD between MBCT and HEP at either 12- or 26-month follow-up. Finally, Farb et al. (2018) compared MBCT to group cognitive therapy (CT), as outlined by Beck, Rush, Shaw, and Emery (1979) and Fava, Rafanelli, Grandi, Conti, and Belluardo (1998). Again, no differences were found between treatment arms in either rates of, or time to, relapse in MDD across 24 months of follow-up. 
Taken together, studies comparing MBCT to an active psychosocial comparator largely fail to demonstrate that MBCT is associated with greater reductions in risk of relapse/recurrence. However, two studies (Meadows et al., 2014; Williams et al., 2014) found MBCT to be superior under certain variations in treatment setting or client characteristics. In addition, two studies utilized active comparators that have, themselves, been the focus of study in the past (Farb et al., 2018; Shallcross et al., 2018). Variability in comparison treatments, settings, and participants may explain the lack of consistent superiority of MBCT. However, variability in some unmeasured mechanisms explain the less than encouraging results when MBCT is compared to an active psychosocial intervention. Decentering is an important mechanism that underpins MBCT theory (Segal, Williams, \& Teasdale, 2013), but has rarely been the focus in the published MBCT literature. Increasing understanding of the role of decentering in MBCT may help to explain for whom, and under what other variations in treatment, time and setting, MBCT is effective in reducing relapse/recurrence in MDD.

\section{Decentering as a candidate therapeutic mechanism of $\mathrm{MBCT}$}

One presumed mechanism associated with MBCT is decentering which is commonly defined as the ability to observe one's thoughts and feelings as temporary, objective events in the mind, as opposed to reflections of the self that are necessarily true (Fresco, Moore et al., 2007; Safran \& Segal, 1990). Bernstein et al. (2015), in their metacognitive processes model of decentering, decomposed decentering into three interrelated psychological processes: metaawareness, disidentification from internal experience, and reduced reactivity to thought content. Meta-awareness is defined as “awareness of present moment experience as a process” (Bernstein et al., 2015, p. 600) and can be thought of as awareness of how someone is thinking (i.e., the process), as contrasted with what they are thinking (i.e., the content). Disidentification from 
internal experience is defined as "the experience of internal states as separate from one’s self” (Bernstein et al., 2015, p. 600). Reduced reactivity to thought content is defined as "the reduced effects of thought content on other mental processes (e.g., attention, emotion, cognitive elaboration, motivation, motor planning)” (Bernstein et al., 2015, p. 601). This model posits that meta-awareness initiates disidentification and reduced reactivity, which both affect one another, and both reinforce meta-awareness. Evidence for this model comes in the form of psychometric studies of measures of decentering, experience sampling studies, behavioral assessments, and experimental inductions and micro-interventions, all of which support the metacognitive processes model (see Bernstein, Hadash, \& Fresco, 2019 for a review). Similarly, additional theoretical accounts (Bendall \& Royle, 2018; Bernstein et al., 2015; King \& Fresco, 2019; David R. Vago \& Silbersweig, 2012) and empirical findings (e.g., Scult et al., 2019) are beginning to reveal the neurobehavioral underpinnings of decentering, suggesting that mindfulness-based interventions (MBIs) lead to engagement and enduring alterations in large-scale neural networks associated with attention, executive control, and self-referential processing (Hasenkamp \& Barsalou, 2012; Tang, Tang, Tang, \& Lewis-Peacock, 2017; D. R. Vago \& Zeidan, 2016). With respect to treatment findings, gains in decentering predict acute and enduring improvement in mood and anxiety disorders treated with CBT (Fresco, Segal, Buis, \& Kennedy, 2007; HayesSkelton \& Lee, 2018), MBIs (Bieling et al., 2012; Farb et al., 2017; Hoge et al., 2015; Segal et al., 2018), as well as mindfulness enriched cognitive-behavioral therapies (Fresco et al., 2017; Hayes-Skelton, Calloway, Roemer, \& Orsillo, 2014; Mennin, Fresco, O'Toole, \& Heimberg, 2018; Mennin, Fresco, Ritter, \& Heimberg, 2015; O’Toole, Renna, Mennin, \& Fresco, in press). In particular, two studies have examined the role of decentering as a mechanism of therapeutic action in MBCT (Bieling et al., 2012; Segal et al., 2018). As a secondary analysis of 
the Segal et al. (2010) parent trial Bieling et al. (2012) found that increases in decentering were greatest for MBCT participants. In addition, increases in decentering were associated with reductions in symptoms of depression (from pre-treatment to six-month follow-up) for participants who received MBCT, but not participants receiving ADM. Segal et al. (2019) compared MBCT to cognitive therapy (CT) and examined improvements in decentering as a predictor of relapse/recurrence of MDD over 24-months of follow-up. They found improvements in decentering were prospectively associated with reductions in risk for relapse/recurrence in MDD irrespective of whether the patient received MBCT or CT.

\section{The Current Study}

The current study sought to address several of the limitations described above by utilizing a three-arm design which compared MBCT against relaxation (RGT), an active comparator matched to MBCT on group, therapist contact and a control condition (TAU). The use of relaxation group therapy is a clinically meaningful comparison group as participants at the beginning of mindfulness training often have difficulty distinguishing between the active nonjudgmental awareness of a mindful state and the sleepy, unaware, nonjudgmental state of deep relaxation (Segal et al., 2013, p. 155). The current study is also noteworthy for directly assessing decentering, a purported therapeutic mechanism in MBCT (Segal et al., 2013, p. 91), allowing for investigation of the role of decentering as a differential mechanism between MBCT, relaxation treatment, and TAU groups.

\section{Hypotheses}

1. MBCT will be associated with equivalent risk of relapse/recurrence of MDD at 12-month follow-up, and equivalent time to relapse/recurrence, as compared to relaxation group therapy or TAU. 
2. MBCT patients as compared to RGT or TAU patients will experience equivalent reductions in depression symptoms during active treatment.

3. Increases in decentering during active treatment will be greater for patients receiving MBCT, as compared to patients receiving relaxation or TAU.

4. Increases in decentering during active treatment will be associated with reductions in relapse/recurrence of MDD for patients receiving MBCT, but not for patients receiving relaxation or TAU.

\section{Method}

\section{Study Design and Participants}

The study design was a randomized (1:1:1 - no blocking or stratification) controlled comparison of MBCT, relaxation group therapy (RGT), or treatment as usual (TAU). A research assistant assigned participants to groups with randomization achieved by computer-generated random number sequencing. Participants were recruited from the Vancouver metropolitan area by newspaper advertisements, postings on community bulletin boards and online platforms (e.g., Craigslist), and referrals from local outpatient mental health clinics in 2008. All potential participants were contacted by a research assistant and completed an initial phone screen. Eligible individuals who agreed to participate, completed an initial testing session lasting 2.5 hrs. During this assessment, participants provided informed consent and were administered the Structured Clinical Interview for the DSM IV (SCID-IV; First, Gibbon, Spitzer, Williams, \& Benjamin, 1997), the Hamilton Rating Scale for Depression (HRSD; Hamilton, 1960), and the $2^{\text {nd }}$ edition of the Beck Depression Inventory (BDI-II; Beck, Steer, \& Brown, 1996).

Inclusion criteria were: (1) a Diagnostic and Statistical Manual of Mental Disorders ( $4^{\text {th }}$ ed.; DSM IV-TR; American Psychiatric Association [APA], diagnosis of MDD without 
psychotic features, in Full Remission; (2) three or more prior MDEs; (3) age between 18 and 65 years; (4) cognitive reactivity (CR) or mood-activated dysfunctional beliefs score greater than or equal to eight (see Supplemental Materials for details); (5) a score of less than 10 on the HRSD; (6) minimum of a 10 week period free of psychotropic medication other than stable dosage of antidepressant medication for a minimum of four weeks; (7) fluency in English; and, (8) ability to give informed consent and complete questionnaires unassisted. Exclusion criteria were: (1) a SCID-IV diagnosis of bipolar disorder (past or present), schizophreniform disorders, substance abuse or dependence (current or within the past six months), borderline or antisocial personality disorder, or neurocognitive disorders; 2) current psychotherapy or counselling more frequently than twice per month; (3) current practice of meditation more than once per week or yoga more than twice per week; (4) electroconvulsive therapy within the past six months; or (5) selfreported ingestion of alcohol or other psychoactive substances within the past 48 hrs.

Of the 468 participants were assessed as part of the telephone screening, 233 (49.79\%) did not meet inclusion criteria (see Fig. 1 for CONSORT diagram of participant flow). The final sample included 227 participants randomized to MBCT $(n=74)$, RGT $(n=77)$, or TAU $(n=76)$. Power analysis (Lachin, 1981) indicated that, for comparison of survival curves between two groups, assuming a hazard ratio of .79 for comparison of MBCT and an active comparator (Kuyken et al., 2016) would require 103 participants per group to achieve $80 \%$ power. This hazard ratio assumes a small difference in survival between MBCT and the comparison condition (as a hazard ratio of 1.0 indicates perfect equivalence in relapse/recurrence). Recruitment methods were approved by the institutional review board of the University of British Columbia at Vancouver. A total of 11 participants who did not meet eligibility criteria (all because they had fewer than three prior MDEs) were randomized to MBCT $(n=2)$, RGT ( $n$ 
$=2$ ), and TAU $(n=4)$ (they initially reported four or more prior MDEs, then indicated fewer on a subsequent assessment). These cases were excluded in all analyses. Results were identical with these cases included or excluded. Results with these cases included are available upon request.

\section{Interventions}

Mindfulness-based Cognitive Therapy. Participants randomized to MBCT received eight-weekly, two-hour sessions (Segal et al., 2013). MBCT combines MBSR meditation practices (e.g., body scan, mindful stretching, mindfulness of breath/body/sounds/thoughts) with traditional CT techniques (e.g., psychoeducation about depression symptoms and automatic thoughts, exercises designed to demonstrate how the nature of one's thoughts change with one's mood, questioning of automatic thoughts and creating a relapse prevention plan). Finally, participants engaged in a daily meditation practice and homework exercises directed at integrating the application of awareness skills into daily life. Each MBCT group was led by a masters-level clinician who was an active MBCT/MBSR instructor.

Active comparison condition (RGT). The revised edition of the Changeways Relaxation Programme (Paterson, 1997) served as the active comparator controlling for non-specific group factors including group participation, expectation of change or therapeutic contact and attention. The rationale was that relaxation can be used to better manage life stressors which precipitate depressive episodes. Participants received eight-weekly, two-hour relaxation training sessions. This group program combines psychoeducation regarding the effects of stress, diaphragmatic breathing, progressive muscle relaxation, passive relaxation and imagery. It also incorporates time for participants to discuss the events of the week to facilitate the supportive aspect of group participation. Finally, participants were asked to engage in daily exercises to practice the various relaxation strategies. Each RGT group was led by a doctoral-level therapist. 
Treatment as usual. Participants randomized to the TAU group were instructed that they would receive MBCT at the end of the follow-up period and to seek help from their family doctors or other sources as the normally would, should they encounter symptomatic deterioration or other difficulties over the course of the study. At the end of the follow-up phase, participants in the TAU and RGT group were offered the opportunity to receive MBCT.

\section{Measures}

Therapist training and fidelity. Therapists’ degree of adherence to the MBCT protocol was monitored using the 17-item MBCT Adherence Scale (MBCT-AS; Segal, Teasdale, Williams, \& Gemar, 2002). A subset of 18 (25\%) MBCT sessions were evaluated by a psychologist and advanced doctoral student who were familiar with MBCT but separate from the research team. Audio tapes were randomly selected from stratified blocks of early $(1,2,3)$, middle $(4,5)$, and late $(6,7,8)$ sessions. Scores were calculated based on a scale ranging from ' 0 ', which indicated “no evidence for item” to “2”, which indicated “definite evidence” for 17 different items delivered across each session. Inter-rater reliability for the MBCT-AS was estimated using the intraclass correlation coefficient (ICC) for all items across two raters and all tapes. This ICC value (.78) indicated good reliability. Mean ratings of therapists indicated an acceptable degree of adherence of the instructors to the MBCT protocol $(M=1.42, \mathrm{SD}=.89)$. Note that adherence is likely underestimated in this sample as items were scored 0 (i.e., no evidence) if they were not present during a session; however, the absence of an item may be appropriate given stage in treatment. For example, in session 1, as participants have yet to be been assigned homework, item 7 “homework review” would be scored '0’.

Treatment adherence for the RGT group was assessed with the eight-item Changeways Relaxation Programme Adherence Scale, which was developed for the current study to measure 
adherence in a manner similar to the MBCT-AS (Segal et al, 2002). A subset of 17 (24\%) RGT sessions were evaluated by the same psychologist and advanced doctoral student. Audio tapes were randomly selected from stratified blocks of early $(1,2,3)$, middle $(4,5)$, and late $(6,7,8)$ sessions. Like the MBCT-AS, scores were calculated based on a scale ranging from ' 0 ', which indicated "no evidence for item" to "2", which indicated “definite evidence” for 17 difference items delivered across each session. The ICC value across two raters (.89) indicated a good degree of inter-rater reliability. Mean ratings of therapists indicated an acceptable degree of adherence of the instructors to the RGT protocol $(M=1.72, \mathrm{SD}=.89)$.

Primary and Secondary Outcomes. The primary outcome was incidence of depression relapse and time to relapse over 12 months of follow-up. DSM-IV-TR criteria for a major depressive episode (APA, 2000) were assessed by the mood disorders module of the SCID-IV (First et al, 1994). Clinical interviews were conducted by a research assistant blind to group assignment. Secondary outcomes were depression symptoms (BDI-II; Beck, Steer, \& Brown, 1996) and decentering measured with Experiences Questionnaire scale (EQ; Fresco, Moore et al., 2007) at baseline, mid-treatment, and immediately following the 8-week intervention phase.

Demographics and clinical variables include age, gender identity, marital status, ethnicity, income, years of education, age at onset of first depressive episode, number of previous depressive episodes, and current medications. All data, study materials, and analysis code is available from the first author, by request.

\section{Results}

\section{Preliminary Analyses}

All analyses were conducted using SPSS, ver. 28.0.0 (IBM Corp, 2021). Descriptive statistics for participants of the three treatment arms are presented in Table 1. At pre-treatment, 
the groups differed significantly on depression symptoms with MBCT participants reporting more symptoms than TAU participants (Cohen's $f=.20$ ); no significant differences were found between MBCT and RGT $(f=.13)$ or between RGT and TAU $(f=.07)$. By mid-treatment, this difference had disappeared. MBCT and RGT also differed on the percentage of participants that attended four or more sessions, with a significantly larger number of MBCT participants reaching this benchmark than RGT participants, suggesting that MBCT was more acceptable.

\section{Missing Data}

Rates of missing data were assessed in all three treatment groups at pre-treatment, posttreatment, and 12-month follow-up assessments. A significant difference was found for completion of the pre- and post-treatment assessments, $\mathrm{X}^{2}(2, N=227)=5.47, p=.065$, and this difference largely stemmed from a higher completion rate in TAU (80\%), compared to both MBCT (74\%) and RGT (64\%), as the comparison between MBCT and RGT was not statistically significant, $\mathrm{X}^{2}(1, N=151)=2.01, p=.16$. With regard to completion of the follow-up assessment, comparison of all three groups was statistically significant, $\mathrm{X}^{2}(2, N=227)=12.38$, $p=.002$, while the comparison of MBCT and RGT just missed this cut-off, $\mathrm{X}^{2}(1, N=151)=$ 3.17, $p=.075$. Completion was highest for TAU participants (79\%), followed by MBCT participants (66\%), and RGT participants (52\%). One possible explanation for higher completion rate for TAU participants may be due to the fact that MBCT treatment was offered to these participants at the end of the follow-up period, acting as an incentive for TAU participants to complete all waves of data collection. Completion of both the pre- and post-treatment assessments was not associated with age, gender, marital status, level of education, number of prior depressive episodes, whether the participant was taking psychotropic medication, or whether the participant relapsed during the 12-month follow-up period (all $p<.11$ ). Similar 
results are obtained when completion of the follow-up assessment was examined. Gender identity was most strongly associated with completion of follow-up ( $p=.13$ ) as a higher percentage of females (69\%) completed this assessment than their male counterparts (59\%).

\section{MDD Relapse/Recurrence}

All analyses were conducted on the intention to treat (ITT) sample and are reported below. Per protocol (PP) analyses included individuals who completed a minimal effective dose of therapy (four or more sessions) are reported in Supplemental Materials. Sample sizes at 12month follow-up were similar between ITT and PP samples (ITT: MBCT: $n=49$, RGT: $n=40$, TAU: $n=60$; PP: MBCT: $n=46$, RGT: $n=32$, TAU: $n=60)$. Comparison of ITT and PP samples on demographic and outcome variables at all assessment points did not reveal any significant differences using a Bonferroni-corrected alpha level of .005 (.05/10). Time to relapse was estimated by means of Cox proportional hazard survival analysis. Post-treatment BDI score and treatment group were both entered as predictors in the same step. For the ITT sample, relapse rates at the 12-month follow-up were 41\% (20/49) in MBCT, 36\% (14/39) in RGT, and 45\% (27/60) in TAU participants. Consistent with Hypothesis 1, differences in these values did not reach statistical significance for the comparison of all three groups, $\mathrm{X}^{2}(d f=2, n=148)=$ $.81, p=.67$. In addition, treatment group was also not a significant predictor of time to relapse, Wald $(1, n=227)=1.23, p=.27$, hazard ratio $=.79\left(95 \%\right.$ confidence interval $\left[\mathrm{CI}_{95 \%}\right]=.52-$ 1.20), also consistent with Hypothesis 1.

\section{Active Treatment}

Repeated-measures ANOVA was used to evaluate change in depression and decentering, from pre- to mid- to post-treatment, as a function of treatment group. In the ITT sample, a significant main effect of time, $F(2,242)=13.29, p<.001, f=.33$, was qualified by a time $\mathrm{x}$ 
treatment group interaction, $F(4,242)=5.98, p<.001, f=.31$. Depression increased from preto mid-treatment in all groups, except MBCT participants (MBCT: $f=.21$; RGT: $f=.53$; TAU: $f$ $=.83$ ) and a significant drop in depression was only observed in the MBCT group (MBCT: $f=$ .66 ; RGT: $f=.22$; TAU: $f=.08$ ), counter to Hypothesis 2. Results using multilevel modeling were substantively identical to those reported above and are reported in Supplemental Materials.

Regarding changes in decentering, in the ITT sample, a significant main effect of time, $F$ $(2,208)=13.62, p<.001, f=.36$, was qualified by a time $\mathrm{x}$ treatment group interaction, $F(4$, $242)=11.83, p<.001, f=.48$. Decentering increased significantly from pre- to mid-treatment for MBCT participants, only $(f=.45)$, did not change for RGT participants $(f=.31)$, and decreased significantly for TAU participants $(f=-.61)$. From mid- to post-treatment, decentering increased significantly in all groups, but the magnitude of this increase was greatest in MBCT participants (MBCT: $f=.71$; RGT: $f=.51$; TAU: $f=.43$ ). Taken together, these results support Hypothesis 3. Results using multilevel modeling were substantively identical and are reported in Supplemental Materials.

\section{Decentering as a Mechanism of Therapeutic Change}

Like the evaluation of Hypothesis 1, post-treatment depression and treatment group were entered as predictors in a first step of a Cox proportional hazard survival analysis. To examine the role of decentering, change in decentering from mid- to post-treatment and its interaction with treatment group were entered in subsequent steps. Change in decentering was operationalized as mid- to post-treatment change for two reasons. First and foremost, change in decentering would be expected to be greater in the second half (i.e., last four sessions) of MBCT treatment as decentering does not become a focal point of sessions until session 6 (Segal et al., 2013). This idea is supported by the fact that substantially more change occurred from mid- to 
post-treatment for MBCT participants in the current study $(f=.69)$ than during the pre- to midtreatment epoch $(f=.50)$. Secondly, this way of representing MBCT-linked change in decentering was also used by Bieling et al. (2012).

The results using treatment group and post-treatment depression as predictors is presented above and so will not be repeated here. In the second step (once change in decentering was added as a predictor), neither treatment group, Wald $(1, n=235)=1.07, p=.30$, hazard ratio $=.80\left(\mathrm{CI}_{95 \%}=.52-1.22\right)$, nor change in decentering were significant predictors of time to relapse, Wald $(1, n=235)=.09, p=.76$, hazard ratio $=1.11\left(\mathrm{CI}_{95 \%}=.56-2.23\right)$. However, once the treatment group $\mathrm{x}$ change in decentering interaction was added as a predictor in the third step, treatment group, Wald $(1, n=235)=6.11, p=.013$, hazard ratio $=.53\left(\mathrm{CI}_{95 \%}=.32-.88\right)$, change in decentering, Wald $(1, n=235)=6.92, p=.009$, hazard ratio $=.14\left(\mathrm{CI}_{95 \%}=.03-.60\right)$, and the interaction of the two, Wald $(1, n=235)=10.08, p=.001$, hazard ratio $=3.43\left(\mathrm{CI}_{95 \%}=\right.$ 1.60 - 7.35), were all statistically significant predictors of time to relapse. We examined the survival curves of each treatment group separately for those participants who either: (1) experienced an increase in decentering from mid- to post-treatment or (2) did not experience such an increase (experienced either no change or a decrease in decentering). This classification corresponded to roughly a median split of participants on mid- to post-treatment change in decentering. The treatment groups experiencing increases in decentering all had lower rates of relapse at 12 months than the groups not experiencing such an increase (see Fig. 2).

To decompose the treatment group $\mathrm{x}$ change in decentering interaction, relapse/recurrence rates at 12-month follow-up were examined per group as a function of whether participants experienced an increase in decentering from mid- to post-treatment. For the ITT sample, relapse/recurrence rates for MBCT participants who experienced an increase in 
decentering, 30\% (6/20), were not significantly lower than MBCT participants who did not experience an increase in decentering, 58\% $(7 / 12), \mathrm{X}^{2}(d f=1, n=32)=2.50, p=.11$. However, this result, $h=.57$, corresponded to Cohen’s (1988) convention for a medium effect. Comparison of participants who experienced increases in decentering, 29\% (4/14), or not within RGT, 22\% (2/9), did not reach statistical significance, $\mathrm{X}^{2}(d f=1, n=23)=.12, p=.74$, and corresponded to a small effect, $h=.16$. Similar results were obtained when participants who experienced an increase in decentering, 41\% (7/17), or not within TAU, 33\% (6/18), were compared, $\mathrm{X}^{2}(d f=1$, $n=35)=.23, p=.63, h=.17$. Taken together, these results suggest that changes in decentering were much more impactful on 12-month relapse/recurrence rates in MBCT, as compared to RGT or TAU, consistent with Hypothesis 4. The lack of statistically significant results reflects a lack of power in subgroups of participants subdivided by change in decentering.

\section{Discussion}

Consistent with expectations, MBCT performed equivalently to RGT with regard to relapse/recurrence rates at 12-month follow-up. This result would seem to be largely driven by the unusually low relapse/recurrence rate for RGT. It is unlikely that this result is due to MBCT lack of fidelity given that MBCT therapists were highly trained and largely adherent. In addition, the 12-month relapse/recurrence rate for MBCT (43\%) is consistent with what has been found in other MBCT trials (Kuyken et al., 2008; 2015; Shallcross et al., 2018; Williams et al., 2014). The relapse/recurrence rate for RGT participants (34\%) is, however, substantially lower than three of the four prior trials comparing MBCT to an active comparator (Meadows et al., 2014: 55\%; Shallcross et al., 2018: 50\%; Williams et al., 2014: 50\%). Given that relaxation is considered by many to be a legitimate intervention and that the form of relaxation administered was, itself, a manualized treatment, these factors might have resulted in an active comparator that was more 
credible and, hence, more effective than active comparators used in past MBCT trials (but see Farb et al. (2018), who used CT as a comparator).

MBCT did not outperform TAU on the same outcome; a result which is inconsistent with much of the prior research comparing MBCT and TAU (Godfrin \& van Heeringen, 2010; Ma \& Teasdale, 2004; Teasdale, Williams, Ridgeway, Soulsby, \& Lau, 2000). This result would seem to be largely driven by the unusually low relapse/recurrence rate for TAU participants in the current trial. Regarding the lack of equivalence with TAU, we note that TAU participants evidenced a lower relapse/recurrence rate (42\%) when compared to other MBCT trials (e.g., the rate for TAU participants in Williams et al. (2014) was 53\% and for TAU participants in Ma \& Teasdale (2004) and Teasdale et al. (2005) was between 60 and 80\%). Results from Segal et al. (2010), found approximately a 60\% relapse/recurrence rate over an equivalent follow-up period for placebo participants. The likely explanation is that TAU participants sought other treatment, which was not prohibited in the current trial. Interestingly, the relapse/recurrence rate for TAU participants in the current trial is closest to the rate found in Bondolfi et al. (2010; 36\%), which compared MBCT plus TAU to TAU alone. Although the MBCT group demonstrated a longer time to relapse/recurrence, the relapse/recurrence rates at the end of the trial did not differentiate the two groups over the 14-month study period. This lack of difference was attributed to the relative ease of access to mental health care in the Swiss healthcare system, where the trial took place, as compared to prior MBCT trials conducted elsewhere.

Consistent with Hypotheses 2 through 4, MBCT patients experienced greater increases in decentering, and greater reductions in depression symptoms, during active treatment as compared to RGT or TAU patients. In addition, these increases in decentering were uniquely associated with reductions in risk of relapse/recurrence for patients who received MBCT, but not 
RGT or TAU. These results are noteworthy because the current study represents only the third trial (Bieling et al., 2012; Segal et al., 2019) to examine the role of decentering in predicting outcome for MBCT with respect to depression recurrence/relapse. The current study also represents the first to identify decentering as a mechanism that is unique to MBCT, when compared to a well-equated psychosocial comparator. However, these results should be considered exploratory in nature given the small cell sizes, particularly among participants who did not experience change in decentering. Hypothesis 3, that change in decentering would be greatest in MBCT, was supported. Hypothesis 4, that change in decentering would be associated with reductions in relapse/recurrence for MBCT, only, was partially supported, as change in decentering was associated with improvement for both MBCT and RGT. Taken together, the results suggest that decentering was a more important factor in predicting 12-month relapse/recurrence of MDD than treatment group (see Fig. 1 and 2) and, furthermore, that decreases in relapse/recurrence depended on increases in decentering, regardless of group assignment. Given the paucity of research on mechanisms and, again, the small cell sizes in the current trial, future studies are clearly needed to attempt to replicate what has been found in the current study. However, the fact that two studies have now identified decentering as a mechanism that is specific to MBCT is encouraging. What remains needed are studies that examine other variables that both predict successful outcome in, and are relatively specific to, MBCT (it seems unlikely that decentering is the only one). One such variable may be cognitive reactivity (CR). The current study sampled participants high in CR, limiting variability and the ability to use CR variability to predict treatment response. Future research should investigate the ability of CR to predict response to MBCT, both as a mechanism and potential variable to identify for whom MBCT may be most beneficial. Additional focus on mechanisms will help to 
refine MBCT and increase its efficiency by potentially removing components of treatment that may not be associated with positive outcome and increasing components that are.

Future research into decentering as a transtheoretical mechanism of change would benefit from multimethod assessment of decentering, including behavioral (Shepherd, Coifman, Matt, \& Fresco, 2016) and physiological assessment. As mentioned above, emerging research is beginning to identify the neurobehavioral underpinnings of decentering (e.g., King \& Fresco, 2019; Scult et al., 2019). Future research is needed to identify if decentering-mediated reductions in relapse/recurrence in MDD are reflected in alterations in large-scale neural networks (e.g., King \& Fresco, 2019).

\section{Clinical Implications}

The implications of the current study, in concert with Bieling and colleagues (2012), indicate the importance of decentering as a potential transdiagnostic mechanism to reduce risk for relapse/recurrence in MDD. Gains in decentering, irrespective of treatment group assignment, predicted likelihood of MDD relapse/recurrence. Although relapse/recurrence rates were equivalent between MBCT and RGT participants, changes in decentering were greater in MBCT, and MBCT participants who exhibited decentering gains evidenced greater reductions in risk for relapse/recurrence of MDD in this group. Though mindfulness and relaxation are conceptually distinct, a point emphasized at the outset of MBCT, in the current trial, both approaches were equally helpful in preventing relapse/recurrence. Interestingly, treatment linked gains in decentering, irrespective of treatment modality reduced recurrence of MDD. Taken together, the results of this trial suggest that whether participants improved in their ability to decenter was at least as important, if not more important, than to which type of treatment they were assigned.

\section{Limitations}


Although the current study has a number of strengths, it is not without limitations. First, the particular kinds of interventions that TAU participants received was not assessed. As a result, we cannot ascertain why TAU participants evidenced a lower rate of relapse/recurrence as compared to prior MBCT trials (42\% vs. 53 to 80\%). Second, the analyses which followed-up the significant interaction effect suffered from low sample size ( $n$ ’s $<10$ in some cells) and inadequate power. This difficulty is often encountered in psychotherapy process research when trying to examine the interactive effects of a treatment with and without a putative mechanism, when therapists are adherent. Consistent with this idea, the groups with the smallest cell sizes in the current trial were MBCT ( $n=12$, for the ITT sample) and RGT ( $n=9$, for the ITT sample) participants who experienced no gains in decentering. Third, participants were required to be in remission from MDD to be admitted to the trial, were not systematically brought to remission with study provided treatment prior to their participation, and how they achieved remission was not assessed. As a result, how participants achieved remission (i.e., psychotherapy, medication, both, neither) remains a potential source of variability in the participants of the current trial. Fourth, participants were selected, in part, based on the cognitive reactivity assessment. This inclusion criterion differentiates the current trial from prior MBCT trials and, insofar as participants were selected to be cognitively reactive, limits its generalizability. Finally, as assessments were conducted at pre-, mid-, and post-treatment during active treatment it is likely that theoretically-relevant change in decentering that occurred during active treatment was missed. It would be reasonable to expect decentering to change on a session-by-session basis, while the span between pre- and mid-treatment, and mid- and post-treatment encompassed four sessions (see Hayes-Skelton \& Lee (2018) for an example with social phobia patients; see O’Toole et al. (2019) for an example with MDD/GAD patients). 


\section{Conclusions}

Findings of the current trial indicate that MBCT was equivalent to RGT and TAU in reducing rates of relapse/recurrence in MDD over 12-months of post-treatment follow-up. However, self-reported decentering emerged as a clinically meaningful mechanism associated with reductions in relapse/recurrence in MDD. In addition, gains in decentering were greatest for MBCT participants, as compared to patients receiving two active comparators: TAU and RGT. Research on the mechanisms of MBCT is still in its infancy and there are a large number of unanswered questions. Future research should attempt to identify modifications of MBCT to maximize increases in decentering, specify which components of decentering are most strongly predictive of outcome in MBCT, and identify other candidate mechanisms of therapeutic action (e.g., reductions in cognitive reactivity or rumination). 
Table 1

Descriptive statistics

\begin{tabular}{|c|c|c|c|c|}
\hline \multirow[b]{2}{*}{ Variable } & \multicolumn{3}{|l|}{ Group } & \multirow[b]{2}{*}{$p$-value, Cohen’s $f$} \\
\hline & $\operatorname{MBCT}(n=74)$ & RGT $(n=77)$ & TAU $(n=76)$ & \\
\hline \multicolumn{5}{|l|}{ Pre-treatment } \\
\hline Gender identity \# (\% female) & $51(68.90)$ & $46(57.90)$ & $52(68.40)$ & $.41, \mathrm{~N} / \mathrm{A}$ \\
\hline Number of prior depressive episodes $M(S D)$ & $7.57(7.66)$ & $8.14(8.81)$ & $7.38(6.04)$ & $.82, .04$ \\
\hline BDI-II $M(S D)$ & $9.26(8.27)$ & $7.28(7.08)$ & $6.24(6.94)$ & $.04, .17$ \\
\hline EQ $M(S D)$ & $2.89(.68)$ & $3.00(.75)$ & $2.95(.55)$ & $.59, .07$ \\
\hline \multicolumn{5}{|l|}{ Mid-treatment } \\
\hline BDI-II $M(S D)$ & $11.81(9.04)$ & $12.30(8.86)$ & $12.04(8.40)$ & $.97,<.001$ \\
\hline BDI-II $M(S D)$ & $6.00(6.60)$ & $9.04(9.48)$ & $12.57(10.01)$ & $<.001, .31$ \\
\hline EQ $M(S D)$ & $3.06(.61)$ & $3.14(.68)$ & $2.85(.71)$ & $<.001, .44$ \\
\hline Per-protocol completion \# (\% attended $\geq 4$ sessions) & $50(67.60)$ & $32(41.60)$ & N/A & $.001^{*}, \mathrm{~N} / \mathrm{A}$ \\
\hline
\end{tabular}

Note: MBCT = Mindfulness-Based Cognitive Therapy; RGT = relaxation group therapy; TAU = treatment as usual; BDI-II = Beck Depression Inventory-II; EQ = Experiences Questionnaire; ${ }^{*}=$ MBCT vs. RGT, only. 
Figure 1. CONSORT Flow Diagram.

Enrollment

\section{Allocation}

468 Assessed for eligibility

233 Excluded

193 Not meeting inclusion criteria

50 Currently depressed

43 Less than 3 lifetime depressive

episodes/ or 2 not within past 5

years

35 Other diagnoses

42 Regular meditation or prior

mindfulness training

15 Other

48 No longer interested/unknown

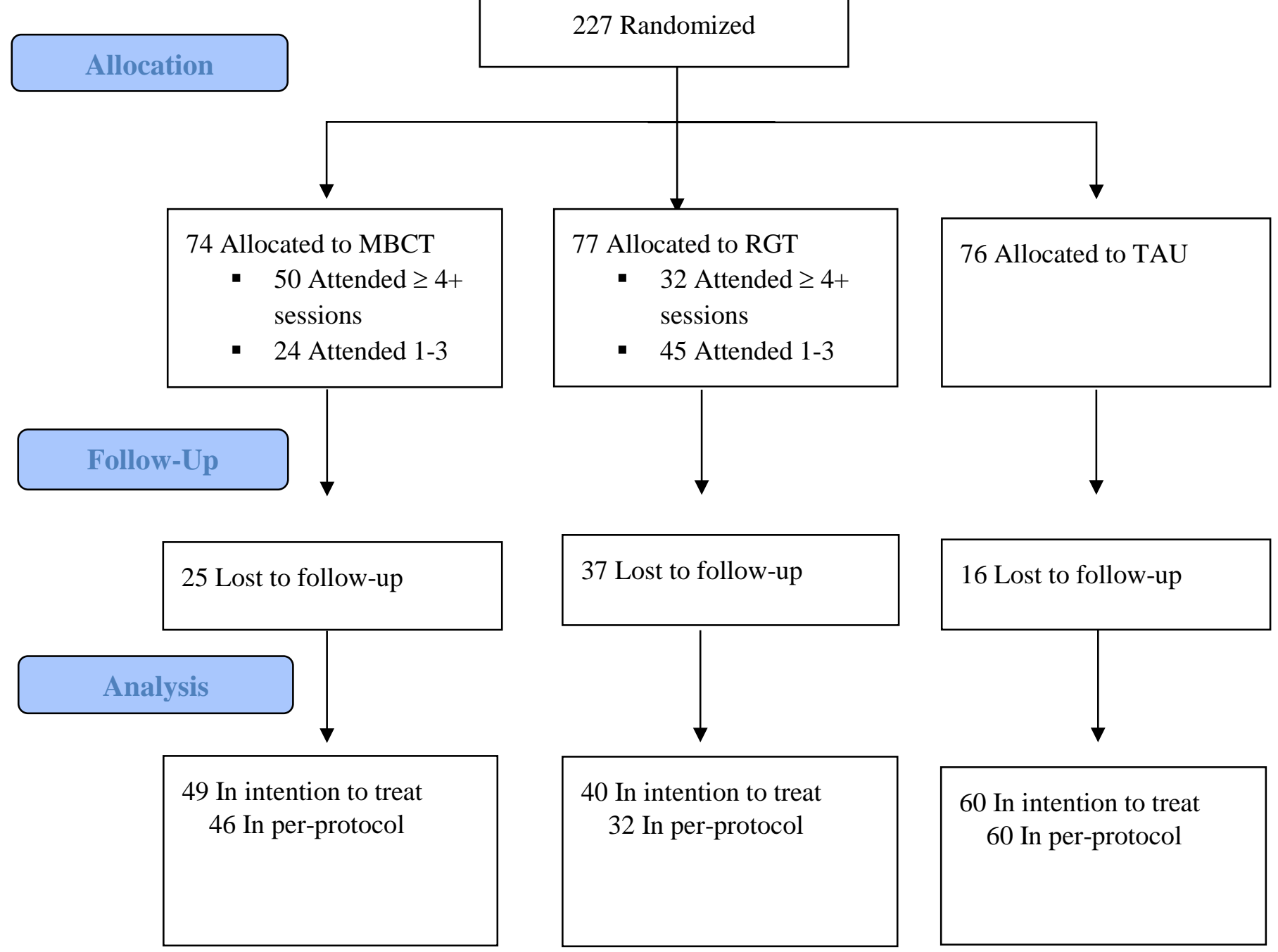


Figure 2. Survival curves showing proportion not relapsing for participants in the Mindfulness-Based Cognitive Therapy (MBCT), relaxation group therapy (RGT), and treatment as usual (TAU) groups as a function of change in decentering in the intent-to-treat (ITT) sample. Note: "Decentering Gains" = Mid- to post-treatment increases in decentering; "Decentering No Gains" = Mid- to posttreatment decreases/no change in decentering.

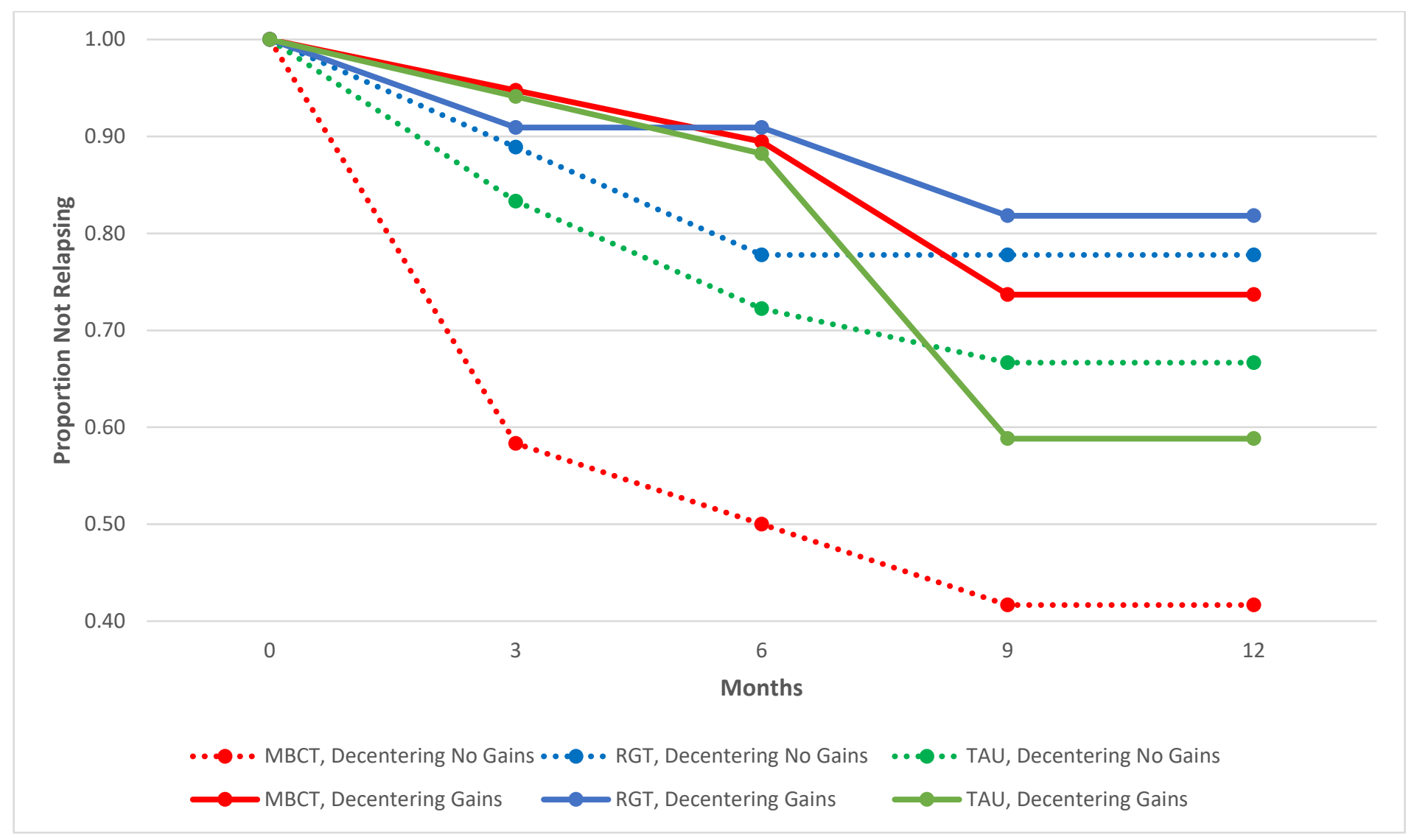




\section{References}

American Psychiatric Association. (2000). Diagnostic and statistical manual of mental disorders: DSM-IV-TR. Washington, DC: American Psychiatric Association.

Barnhofer, T., Reess, T. J., Fissler, M., Winnebeck, M., Grimm, S., Gärtner, M., Fan, Y., Huntenburg, J. M., Schroeter, T. A., Gummersbach, M., Bajbouj, M., \& Hözel, B. K. (2021). Effects of mindfulness training on emotion regulation in patients with depression: Reduced dorsolateral prefrontal cortex activation indexes early beneficial changes. Psychosomatic Medicine, 83, 579-591

Beck, A. T., Steer, R. A., \& Brown, G. (1996). Beck Depression Inventory-II. Psychological Assessment.

Beck, A. T., Rush, A. J., Shaw, B. F., \& Emery, G. (1979). Cognitive Therapy of Depression. New York: Guilford Press.

Bendall, R. C. A., \& Royle, S. (2018). Decentering mediates the relationship between vmPFC activation during a stressor and positive emotion during stress recovery. Journal of Neurophysiology, 120, 2379-2382. doi:10.1152/jn.00353.2018

Bernstein, A., Hadash, Y., \& Fresco, D. M. (2019). Metacognitive processes model of decentering: emerging methods and insights. Current Opinion in Psychology, 28, 245251. https://doi.org/10.1016/j.copsyc.2019.01.019

Bernstein, A., Hadash, Y., Lichtash, Y., Tanay, G., Shepherd, K., \& Fresco, D. M. (2015). Decentering and related constructs: A critical review and metacognitive processes model. Perspectives on Psychological Science, 10, 599-617. doi:10.1177/1745691615594577

Bieling, P. J., Hawley, L. L., Bloch, R. T., Corcoran, K. M., Levitan, R. D., Young, T. L., Macqueen, G. M., \& Segal, Z. V. (2012). Treatment-specific changes in decentering 
following mindfulness-based cognitive therapy versus antidepressant medication or placebo for prevention of depressive relapse. Journal of Consulting and Clinical Psychology, 80, 365-372. doi:10.1037/a0027483

Bondolfi, G., Jermann, F., der Linden, M. V., Gex-Fabry, M., Bizzini, L., Rouget, B. W., MyersArrazola, L., Gonzalez, C., Segal, Z., Aubry, J. M., \& Bertschy, G. (2010). Depression relapse prophylaxis with Mindfulness-Based Cognitive Therapy: Replication and extension in the Swiss health care system. Journal of Affective Disorders, 122, 224-231. https://doi.org/10.1016/j.jad.2009.07.007

Cella, D. F., \& Perry, S. W. (1986). Reliability and concurrent validity of three visual-analogue mood scales. Psychological Reports, 59, 827-833.

https://doi.org/10.2466/pr0.1986.59.2.827

Clark, D. M., \& Teasdale, J. D. (1985). Constraints on the effects of mood on memory. Journal of Personality and Social Psychology, 48, 1595-1608. https://doi.org/10.1037/00223514.48.6.1595

Cohen, J. (1988). Statistical Power Analysis for the Behavioral Sciences (2nd ed.). Hillsdale, NJ: Lawrence Erlbaum Associates, Publishers.

Cuijpers, P., Berking, M., Andersson, G., Quigley, L., Kleiboer, A., \& Dobson, K. S. (2013). A meta-analysis of cognitive-behavioural therapy for adult depression, alone and in comparison with other treatments. Canadian Journal of Psychiatry. Revue Canadienne de Psychiatrie, 58, 376-385. https://doi.org/10.1177/070674371305800702

Cuijpers, P., Noma, H., Karyotaki, E., Cipriani, A., \& Furukawa, T. A. (2019). Effectiveness and acceptability of cognitive behavior therapy delivery formats in adults with depression: A 
network meta-analysis. JAMA Psychiatry, 76, 700-707.

https://doi.org/10.1001/jamapsychiatry.2019.0268

Eaton, W. W., Shao, H., Nestadt, G., Lee, H. B., Bienvenu, O. J., \& Zandi, P. (2008).

Population-based study of first onset and chronicity in major depressive

disorder. Archives of General Psychiatry, 65, 513-520.

https://doi.org/10.1001/archpsyc.65.5.513

Farb, N., Anderson, A., Ravindran, A., Hawley, L., Irving, J., Mancuso, E., Gulamani, T., Williams, G., Ferguson, A., \& Segal, Z. V. (2018). Prevention of relapse/recurrence in major depressive disorder with either Mindfulness-Based Cognitive Therapy or Cognitive Therapy. Journal of Consulting and Clinical Psychology, 86, 200-204. doi:10.1037/сcp0000266

Fava, G. A., Rafanelli, C., Grandi, S., Conti, S., \& Belluardo, P. (1998). Prevention of recurrent depression with cognitive behavioral therapy: Preliminary findings. Archives of General Psychiatry, 55, 816-820. https://doi.org/10.1001/archpsyc.55.9.816

First, M. B., Gibbon, M., Spitzer, R. L., Williams, J. B. W., \& Benjamin, L. S. (1997).

Structured Clinical Interview for DSM-IV Axis II Personality Disorders (SCID-II). Washington, DC: American Psychiatric Press, Inc.

Fresco, D. M., Moore, M. T., van Dulmen, M., Segal, Z. V., Teasdale, J. D., Ma, H., \& Williams, J. M. G. (2007). Initial psychometric properties of the Experiences Questionnaire. Behavior Therapy, 38, 234-246.

Fresco, D. M., Roy, A. K., Adelsberg, S., Seeley, S., García-Lesy, E., Liston, C., \& Mennin, D. S. (2017). Distinct functional connectivities predict clinical response with Emotion 
Regulation Therapy. Frontiers in Human Neuroscience, 11, 86. doi:10.3389/fnhum.2017.00086

Fresco, D. M., Segal, Z. V., Buis, T., \& Kennedy, S. (2007). Relationship of posttreatment decentering and cognitive reactivity to relapse in major depression. Journal of Consulting and Clinical Psychology, 75, 447-455. doi:10.1037/0022-006x.75.3.447 PMID 17563161

Godfrin, K. A., \& van Heeringen, C. (2010). The effects of Mindfulness-Based Cognitive Therapy on recurrence of depressive episodes, mental health and quality of life: A randomized controlled study. Behaviour Research and Therapy, 48, 738-746. https://doi.org/10.1016/j.brat.2010.04.006

Greenberg, P. E., Stiglin, L. E., Finkelstein, S. N., \& Berndt, E. R. (1993). Depression: A neglected major illness. The Journal of Clinical Psychiatry, 54, 419-424.

Grossberg, J. M., \& Grant, B. F. (1978). Clinical psychophysics: Applications of ratio scaling and signal detection methods to research on pain, fear, drugs, and medical decision making. Psychological Bulletin, 85, 1154-1176.

Hamilton, M. (1960). A rating scale for depression. Journal of Neurology, Neurosurgery, and Psychiatry, 23, 56-62. https://doi.org/10.1136/jnnp.23.1.56

Hasenkamp, W., \& Barsalou, L. W. (2012). Effects of meditation experience on functional connectivity of distributed brain networks. Frontiers in Human Neuroscience, 6, 38. doi:10.3389/fnhum.2012.00038

Hayes-Skelton, S. A., Calloway, A., Roemer, L., \& Orsillo, S. M. (2014). Decentering as a potential common mechanism across two therapies for generalized anxiety disorder. Journal of Consulting and Clinical Psychology, 83, 395-404. doi:10.1037/a0038305 
Hayes-Skelton, S. A., \& Lee, C. S. (2018). Changes in decentering across cognitive behavioral group therapy for social anxiety sisorder. Behavior Therapy, 49, 809-822. doi:10.1016/j.beth.2018.01.005

Hoge, E. A., Bui, E., Goetter, E., Robinaugh, D. J., Ojserkis, R. A., Fresco, D. M., \& Simon, N. M. (2015). Change in decentering mediates improvement in anxiety in MindfulnessBased Stress Reduction for generalized anxiety disorder. Cognitive Therapy and Research, 39, 228-235. doi:10.1007/s10608-014-9646-4

Kessler, R. C., Berglund, P., Demler, O., Jin, R., Koretz, D., Merikangas, K. R., et al. (2003). The epidemiology of major depressive disorder: Results from the National Comorbidity Survey Replication (NCS-R). Journal of the American Medical Association, 289, 30954105.

King, A. P., \& Fresco, D. M. (2019). A neurobehavioral account for decentering as the salve for the distressed mind. Current Opinion of Psychology, 28, 285-293. doi:10.1016/j.copsyc.2019.02.009

Kuyken, W., Byford, S., Taylor, R. S., Watkins, E., Holden, E., White, K., Barrett, B., Byng, R., Evans, A., Mullan, E., \& Teasdale, J. D. (2008). Mindfulness-based cognitive therapy to prevent relapse in recurrent depression. Journal of Consulting and Clinical Psychology, 76, 966-978. https://doi.org/10.1037/a0013786

Kuyken, W., Warren, F. C., Taylor, R. S., Whalley, B., Crane, C., Bondolfi, G., Hayes, R., Huijbers, M., Ma, H., Schweizer, S., Segal, Z., Speckens, A., Teasdale, J. D., Van Heeringen, K., Williams, M., Byford, S., Byng, R., \& Dalgleish, T. (2016). Efficacy of Mindfulness-Based Cognitive Therapy in prevention of depressive relapse: An individual 
patient data meta-analysis from randomized trials. JAMA Psychiatry, 73, 565-574. https://doi.org/10.1001/jamapsychiatry.2016.0076

Lachin J. M. (1981). Introduction to sample size determination and power analysis for clinical trials. Controlled Clinical Trials, 2, 93-113. https://doi.org/10.1016/01972456(81)90001-5

Ma, S. H., \& Teasdale, J. D. (2004). Mindfulness-based cognitive therapy for depression: replication and exploration of differential relapse prevention effects. Journal of Consulting and Clinical Psychology, 72, 31-40. https://doi.org/10.1037/0022006X.72.1.31

Martin, M. (1990). On the induction of mood. Clinical Psychology Review, 10, 669697. https://doi.org/10.1016/0272-7358(90)90075-L

MacCoon, D. G., Imel, Z. E., Rosenkranz, M. A., Sheftel, J. G., Weng, H. Y., Sullivan, J. C., Bonus, K. A., Stoney, C. M., Salomons, T. V., Davidson, R. J., \& Lutz, A. (2012). The validation of an active control intervention for Mindfulness Based Stress Reduction (MBSR). Behaviour Research and Therapy, 50, 3-12. https://doi.org/10.1016/j.brat.2011.10.011

Meadows, G. N., Shawyer, F., Enticott, J. C., Graham, A. L., Judd, F., Martin, P. R., Piterman, L., \& Segal, Z. (2014). Mindfulness-based cognitive therapy for recurrent depression: A translational research study with 2-year follow-up. The Australian and New Zealand Journal of Psychiatry, 48, 743-755. https://doi.org/10.1177/0004867414525841

Mennin, D. S., Fresco, D. M., O'Toole, M. S., \& Heimberg, R. G. (2018). A randomized controlled trial of Emotion Regulation Therapy for generalized anxiety disorder with and 
without co-occurring depression. Journal of Consulting and Clinical Psychology, 86, 268-281. doi:10.1037/сcp0000289

Mennin, D. S., Fresco, D. M., Ritter, M., \& Heimberg, R. G. (2015). An open trial of Emotion Regulation Therapy for generalized anxiety disorder with co-occurring depression. Depression and Anxiety, 32, 614-623. doi:10.1002/da.22377

Mueller, T. I., Leon, A. C., Keller, M. B., Solomon, D. A., Endicott, J., Coryell, W., Warshaw, M., \& Maser, J. D. (1999). Recurrence after recovery from major depressive disorder during 15 years of observational follow-up. The American Journal of Psychiatry, 156, 1000-1006. https://doi.org/10.1176/ajp.156.7.1000

O’Toole, M. S., Renna, M. E., Mennin, D. S., \& Fresco, D. M. (2019). Changes in decentering and reappraisal temporally precede symptom reduction during Emotion Regulation Therapy for generalized anxiety disorder with and without co-occurring depression. Behavior Therapy, 50, 1042-1052. doi:10.1016/j.beth.2018.12.005

Paterson, R. (1997). Changeways: Relaxation Programme. Vancouver, BC: Changeways Clinic.

Piet, J., \& Hougaard, E. (2011). The effect of mindfulness-based cognitive therapy for prevention of relapse in recurrent major depressive disorder: A systematic review and meta-analysis. Clinical Psychology Review, 31, 1032-1040. https://doi.org/10.1016/j.cpr.2011.05.002

Safran, J. D., \& Segal, Z. V. (1990). Interpersonal process in cognitive therapy. Jason Aronson. Scult, M. A., Fresco, D. M., Gunning, F. M., Liston, C., Seeley, S. H., Garcia, E., \& Mennin, D. S. (2019). Changes in functional connectivity following treatment with Emotion Regulation Therapy. Frontiers in Behavioral Neuroscience, 13, 10. https://doi.org/10.3389/fnbeh.2019.00010 
Segal, Z. V., Anderson, A. K., Gulamani, T., Williams, L.-A. D., Desormeau, P., Ferguson, A., Walsh, K., \& Farb, N. A. S. (2019). Practice of therapy acquired regulatory skills and depressive relapse/recurrence prophylaxis following cognitive therapy or mindfulness based cognitive therapy. Journal of Consulting and Clinical Psychology, 87, 161-170. doi:10.1037/сcp0000351

Segal, Z. V., Bieling, P., Young, T., MacQueen, G., Cooke, R., Martin, L., Bloch, R., \& Levitan, R. D. (2010). Antidepressant monotherapy vs sequential pharmacotherapy and mindfulness-based cognitive therapy, or placebo, for relapse prophylaxis in recurrent depression. Archives of General Psychiatry, 67, 1256-1264. https://doi.org/10.1001/archgenpsychiatry.2010.168

Segal, Z. V., Gemar, M., \& Williams, S. (1999). Differential cognitive response to a mood challenge following successful cognitive therapy or pharmacotherapy for unipolar depression. Journal of Abnormal Psychology, 108, 3-10. https://doi.org/10.1037//0021843x.108.1.3

Segal, Z. V., Kennedy, S., Gemar, M., Hood, K., Pedersen, R., \& Buis, T. (2006). Cognitive reactivity to sad mood provocation and the prediction of depressive relapse. Archives of General Psychiatry, 7, 749-755. doi:10.1001/archpsyc.63.7.749

Segal, Z. V., Williams, J. M. G., \& Teasdale, J. D. (2013). Mindfulness-based cognitive therapy for depression (2nd ed.). The Guilford Press.

Segal Z. V., Teasdale J. D., Williams J. M., \& Gemar M. C. (2002). The Mindfulness-Based Cognitive Therapy Adherence Scale: Interrater reliability, adherence to protocol and treatment distinctiveness. Clinical Psychology and Psychotherapy, 9, 131-138. 
Shallcross, A. J., Wilroth, E. C., Fisher, A., Dimidjian, S., Gross, J. J., Visvanathan, P. D., \& Mauss, I. B. (2018). Relapse/Recurrence Prevention in major depressive disorder: 26Month follow-up of Mindfulness-Based Cognitive Therapy versus and active control. Behavior Therapy, 49, 836-849.

Shepherd, K. A., Coifman, K. G., Matt, L. M., \& Fresco, D. M. (2016). Development of a selfdistancing task and initial validation of responses. Psychological Assessment, 28, 841855. https://doi.org/10.1037/pas0000297

Sim, K., Lau, W. K., Sim, J., Sum, M. Y., \& Baldessarini, R. J. (2015). Prevention of relapse and recurrence in adults with major depressive disorder: Systematic review and meta-analyses of controlled trials. The International Journal of Neuropsychopharmacology, 19, pyv076. https://doi.org/10.1093/ijnp/pyv076

Stewart, W.F., Ricci, J.A., Chee, E., Hahn, S.R., \& Morganstein, D. (2003) Cost of lost productive work time among US workers with depression. JAMA, 289, 3135-3144. doi:10.1001/jama.289.23.3135

Tang, Y. Y., Tang, Y., Tang, R., \& Lewis-Peacock, J. A. (2017). Brief mental training reorganizes large-scale brain networks. Frontiers in Systems Neuroscience, 11, 6. doi:10.3389/fnsys.2017.00006

Teasdale, J. D., Segal, Z. V., Williams, J. M., Ridgeway, V. A., Soulsby, J. M., \& Lau, M. A. (2000). Prevention of relapse/recurrence in major depression by mindfulness-based cognitive therapy. Journal of Consulting and Clinical Psychology, 68, 615-623. https://doi.org/10.1037//0022-006x.68.4.615 
Thimm, J. C. \& Johnsen, T. J. (2020). Time trends in the effects of mindfulness-based cognitive therapy for depression: A meta-analysis. Scandinavian Journal of Psychology, 61, 582- 591.

Vago, D. R., \& Silbersweig, D. A. (2012). Self-awareness, self-regulation, and selftranscendence (S-ART): A framework for understanding the neurobiological mechanisms of mindfulness. Frontiers in Human Neuroscience, 6, 296.

doi:10.3389/fnhum.2012.00296

Vago, D. R., \& Zeidan, F. (2016). The brain on silent: mind wandering, mindful awareness, and states of mental tranquility. Annals of the New York Academy of Science, 1373, 96-113. doi:10.1111/nyas.13171

Weinberger, A. H., Gbedemah, M., Martinez, A. M., Nash, D., Galea, S., \& Goodwin, R. D. (2018). Trends in depression prevalence in the USA from 2005 to 2015: Widening disparities in vulnerable groups. Psychological Medicine, 48, 1308-1315. https://doi.org/10.1017/S0033291717002781

Weissman, A. N. (1979). The Dysfunctional Attitude Scale: A validation study. Dissertation Abstracts International, 40(3-B), 1389-1390.

Williams, J. M., Crane, C., Barnhofer, T., Brennan, K., Duggan, D. S., Fennell, M. J., Hackmann, A., Krusche, A., Muse, K., Von Rohr, I. R., Shah, D., Crane, R. S., Eames, C., Jones, M., Radford, S., Silverton, S., Sun, Y., Weatherley-Jones, E., Whitaker, C. J., Russell, D., ... Russell, I. T. (2014). Mindfulness-based cognitive therapy for preventing relapse in recurrent depression: a randomized dismantling trial. Journal of Consulting and Clinical Psychology, 82, 275-286. https://doi.org/10.1037/a0035036 


\section{Supplemental Material}

Cognitive Reactivity (CR) Assessment. Eligible individuals participated in a procedure to assess CR which involved completing one of the two equivalent forms of the 40-item Dysfunctional Attitudes Scale (DAS; Weissman, 1979). Respondents rate their beliefs experienced most of the time on a scale of 1 to 4 , with 1 indicating that they totally agree with the statement and 4 indicating that they totally disagree with the statement. The presentation order (e.g., Form A or B) was counterbalanced across patients. Prior to completing the mood induction, participants provided a brief rating of their mood using the Visual Analogue Scale (VAS; Cella \& Perry, 1986). The VAS is a $100 \mathrm{~mm}$. line anchored by the descriptor word 'sad' located to the left of center and 'happy' located to the right with arrows indicating increasing strength of mood associated with greater distance from center (Grossberg \& Grant, 1978). Participants indicated their current mood by placing a mark on the line between the two endpoints.

Potential participants completed a mood induction where they listened to a digitally remastered, half-speed, non-lyrical piece of classical music presented on a compact disc player and tried to recall a time in their lives when they felt sad. The music was chosen based on previous work by Clark and Teasdale (1985) and is the seven-minute orchestral introduction by Prokofiev entitled "Russia under the Mongolian Yoke." This type of mood induction procedure (i.e., music plus autobiographical recall) has been validated by previous research (Martin, 1990) and is used extensively in CR research (e.g., Segal et al., 1999; 2006). Immediately following the mood induction, participants provided another VAS mood rating, completed the alternate form of the DAS and then provide a final VAS mood rating. Only participants who demonstrated an increased CR score of eight points or more were eligible to participate in the study.

\section{MDD Relapse/Recurrence}


For the PP sample, relapse rates were 40\% (19/47) for MBCT, 24\% (8/33) in RGT, and 42\% (27/64) in WL participants. This difference also did not reach statistical significance for the comparison of all three groups, $\mathrm{X}^{2}(d f=2, n=144)=3.25, p=.20$. Similar to the results in the ITT sample, treatment group was not a significant predictor of time to relapse in the PP sample, Wald $(1, n=165)=.84, p=.36$, hazard ratio $=.81\left(\mathrm{CI}_{95 \%}=.52-1.26\right)$, consistent with Hypothesis 1.

\section{Active Treatment}

In the ITT sample, multilevel modeling (MLM) was also used to examine change in BDIII scores during acute treatment. Both time (pre-, mid-, and post-treatment) and group were estimated as fixed effects, given that sample size and groups per nesting unit were both small (see Kerkhoff \& Nussbeck, 2019 for a review). Similar to the ANOVA results, a significant time $\mathrm{x}$ group interaction was found, $F(4,133.16)=8.23, p<.001$.

In the PP sample, a significant time x group interaction, $F(4,216)=5.27, p=.001, f=$ .31, was found. BDI-II total scores increased significantly from pre- to mid-treatment in the WL group, only (MBCT: $f=.31$ RGT: $f=.37 ; \mathrm{WL}: f=.83$ ) and decreased significantly from mid- to post-treatment in MBCT participants, only (MBCT: $f=.66$; RGT: $f=.19$; WL: $f=.08$ ). Substantively similar results were found using MLM, $F(4,159.77)=8.49, p<.001$.

With regard to EQ scores in the PP sample, a significant time $\mathrm{x}$ group interaction, $F$ (4, $186)=10.11, p<.001, f=.47$, was found. EQ total scores increased significantly from both preto mid-treatment and mid- to post-treatment in MBCT participants, only (pre- to mid: $f=.50$; mid- to post-treatment: $f=.69$ ). EQ scores did not significantly change from either pre- to midtreatment $(f=-.19)$ or mid- to post-treatment $(f=.38)$ in RGT participants, despite mid- to post- 
treatment increases corresponding to a large effect. For WL participants, EQ scores decreased significantly from pre- to mid-treatment $(f=-.61)$ and increased significantly from mid- to posttreatment $(f=.43)$. However, the magnitude of this increase was smaller than that observed in MBCT participants during the same period. Results examining change in EQ scores as a function of group using MLM were substantively identical, as the time x group interactions were significant in both the ITT, $F(4,130.59)=9.71, p<.001$, and PP samples, $F(4,199.18)=9.20$, $p<.001$

\section{Decentering as a Mechanism of Therapeutic Change}

In the PP sample, neither treatment group, Wald $(1, n=165)=.79, p=.38$, hazard ratio $=$ $.82\left(\mathrm{CI}_{95 \%}=.52-1.28\right)$, nor change in decentering were significant predictors of time to relapse in the second step, Wald $(1, n=165)=.02, p=.90$, hazard ratio $=1.05\left(\mathrm{CI}_{95 \%}=.51-2.17\right)$. However, in the third step, treatment group, Wald $(1, n=165)=4.99, p=.026$, hazard ratio $=$ $.56\left(\mathrm{CI}_{95 \%}=.34-.93\right)$, change in decentering, Wald $(1, n=165)=7.03, p=.008$, hazard ratio $=$ $.12\left(\mathrm{CI}_{95 \%}=.03-.58\right)$, and the interaction of the two, Wald $(1, n=165)=9.74, p=.002$, hazard ratio $=3.49\left(\mathrm{CI}_{95 \%}=1.59-7.64\right)$, were all statistically significant predictors of time to relapse. Similar to the results for the ITT sample, the treatment groups experiencing increases in decentering all had lower rates of relapse at 12 months than the groups not experiencing such an increase (see Fig. 1). The rate of relapse/recurrence in MBCT participants who experienced an increase in decentering, 26\% (5/19), did not differ significantly from participants who did not experience an increase, 58\% (7/12), $\mathrm{X}^{2}(d f=1, n=31)=3.18, p=.075$. However, this result, $h=$ .66, exceeded Cohen’s (1988) convention for a medium effect. For RGT participants, relapse/recurrence rates did not differ between those who experienced an increase in decentering, $18 \%(2 / 11)$, and those who did not, 22\% (2/9), $\mathrm{X}^{2}(d f=1, n=20)=.05, p=.82, h=.10$. For WL 
participants, relapse/recurrence rates also did not differ between those who experienced an increase in decentering, 41\% (7/17), and those who did not, 33\% (6/18), $\mathrm{X}^{2}(d f=1, n=35)=$ $.23, p=.63, h=.17$. 
Figure 1. Survival curves showing proportion not relapsing for participants in the MindfulnessBased Cognitive Therapy (MBCT), relaxation group therapy (RGT), and wait list (WL) groups as a function of change in decentering in the per protocol (PP) sample. Note: "EQ Inc" = Mid- to post-treatment increases in decentering; "EQ No Inc” = Mid- to post-treatment decreases/no change in decentering.

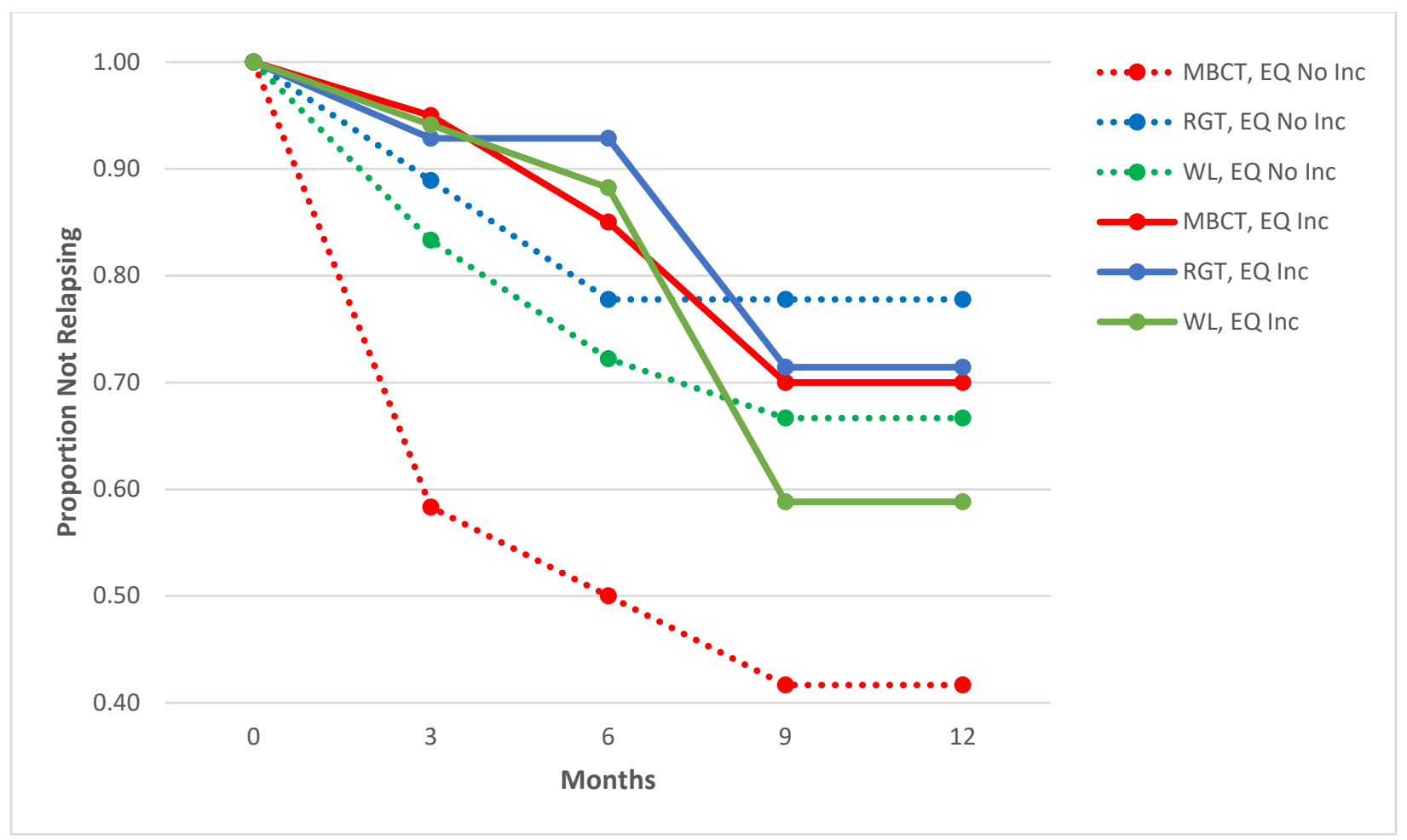

